\title{
Is optical coherence tomography angiography a useful tool in the screening of hydroxychloroquine retinopathy?
}

\author{
Hatice Nur Tarakcioglu • Abdullah Ozkaya (D) Ulviye Yigit
}

Received: 27 April 2020/ Accepted: 16 August 2020/Published online: 27 August 2020

(C) Springer Nature B.V. 2020

\begin{abstract}
Purpose To detect the early changes in retinal vasculature via optical coherence tomography angiography (OCTA) by comparing the quantitative OCTA parameters in the group of patients who were using hydroxychloroquine (HCQ) or not.

Methods This was a cross-sectional, comparative, and observational study. The patients who were newly or previously prescribed HCQ for an autoimmune disease were included. OCTA imaging was performed via OCT RT XR Avanti with AngioVue software (Optivue Inc, Freemont, CA). The study group had two groups: a control group (patients newly diagnosed and who were not taking any medication) and a treatment group (patients who were receiving HCQ
\end{abstract}

H. N. Tarakcioglu · U. Yigit

Department of Ophthalmology, University of Health

Sciences Bakirkoy Training and Research Hospital,

Istanbul, Turkey

e-mail: h.k.t.1984@hotmail.com

U. Yigit

e-mail: ulviyeyigit@hotmail.com

A. Ozkaya ( $ه)$

Department of Ophthalmology, Memorial Sisli Hospital, Okmeydani, Sisli, 34000 Istanbul, Turkey

e-mail: abdozkaya@gmail.com

\section{A. Ozkaya}

Department of Ophthalmology, Istanbul Aydin

University, Istanbul, Turkey treatment). The main outcome measure was OCTA parameters.

Results A total of 102 eyes of 102 patients were included. There were 70 patients in the treatment group and 32 patients in the control group. All of the vascular density values were similar between the control and treatment groups $(p>0.05$ for all). However, the superficial whole thickness, superficial parafoveal thickness, superficial perifoveal thickness, deep whole thickness, deep parafoveal thickness, and deep perifoveal thickness were thinner in the treatment group than the control group ( $p<0.05$ for all).

Conclusion Vascular density parameters did not differ between the control and treatment groups. However, the retinal thickness values were lower in the treatment group.

Keywords Angiography · Hydroxychloroquine · Optical coherence tomography $\cdot$ Retinopathy

\section{Introduction}

Chloroquines which are originally antimalarial drugs are now being widely used in the treatment of autoimmune diseases [1,2]. Also, they are used as emerging treatment options for skin, oncologic, and pediatric inflammatory disorders [3]. Surprisingly, chloroquines are now widely used as a first-line drug 
in the treatment of-new coronavirus infectionCOVID-19 [4]. The first drug of the group was chloroquine, and less toxic variant hydroxychloroquine (HCQ) was developed later [3, 5]. Chloroquines have toxic effects on retina and eventually lead to socalled bull's eye maculopathy [3]. The optimum safe dose of the drug is still debatable. Traditionally HCQ was used based on ideal body weight (IBW); however, it was reported that in some cases, actual body weight (ABW) might be better to use. The doses of $<5 \mathrm{mg} /$ $\mathrm{kg} /$ day were considered safe $[3,5]$. The duration of HCQ use is another important factor for toxicity. In a large epidemiologic study of 2361 patients who used HCQ for $>5$ years, the HCQ toxicity was reported to be $7.5 \%$ after 5 years of HCQ use and $20 \%$ after 20 years of HCQ use [6]. The exact mechanism of retinal toxicity was not exactly revealed [3]. Toxic effects on retinal pigment epithelium (RPE) cells, accumulation of HCQ in pigmented ocular tissues, and degeneration of photoreceptor cells are among the most possible mechanisms underlying HCQ toxicity $[3,7,8]$. Different screening protocols were described in order to detect HCQ toxicity [3, 9, 10]. Screening is recommended in the first year and annually thereafter [3]. The 10-2 visual field testing, spectral domain optical coherence tomography (OCT), and autofluorescence (AF) imaging are accepted as the main screening tools [3]. In some special circumstances, multifocal electroretinography (mfERG) might be essential also [11, 12]. Many studies were conducted on the sensitivity and specificity of the mentioned tests. Combination of mfERG with either 10-2 visual field testing or OCT was reported as the most reliable screening method [3]. The specificity of 10-2 visual field testing alone was reported as $85.7 \%$, and OCT alone was $78.6 \%$ [3]. Optical coherence tomography angiography (OCTA) is a new noninvasive tool which can assess the retinal vascular system quantitatively, and the efficacy of OCTA in HCQ retinopathy was previously evaluated by several authors [13-16]. In this study, we aimed to detect the early changes in retinal vasculature via OCTA by comparing the quantitative OCTA parameters in patients who were using HCQ or not.

\section{Materials and methods}

This prospective, cross-sectional, and comparative study was conducted in Bakirkoy Training and Research Hospital. A written informed consent for the treatment was obtained from all of the participants. The study adhered to the tenets of the Declaration of Helsinki and local ethics board approval was obtained.

\section{Patients}

The patients who were newly or previously prescribed HCQ for an autoimmune disease were included in the study. Only one eye of each patient was included and the right eye was designated as the study eye in the patients with an even birth month number and the left eye was selected in the patients with an odd birth month number. The patients who had amblyopia or, a retinal disease or, intraocular inflammation or, media opacity or, a history of systemic conditions that could alter the microvasculature (including diabetes, hypertension, cardiovascular disease, and renal disease) were not included.

\section{Examinations}

All of the patients underwent a complete ophthalmological examination, including best-corrected visual acuity (BVCA), slit-lamp biomicroscopy, dilated fundus examination. Optical coherence tomography imaging was performed via OCT RT XR Avanti with AngioVue software (Optivue Inc, Freemont, CA). Structural OCT parameters and OCTA parameters for fovea were analyzed. $6 \times 6$ scan size was used for OCTA imaging, all of the scans were automatically segmented, and the quantitative values that are FAZ area, FAZ perimeter, flow area, vessel density, and retinal thickness were all calculated via the built-in automated software of the device. All measurements were obtained between 9 a.m. and 11 a.m. as the choriocapillary flow might change diurnally [17]. The scans with a signal strength index of $<40$ with motion artifacts and segmentation errors were accepted to have a poor quality and were not analyzed.

Groups and outcomes measures

Firstly, the study group was divided into two groups: a control group (patients newly diagnosed and who were 
not taking any medication) and a treatment group (patients who were receiving HCQ treatment). Also, the treatment group was divided into two subgroups for further analysis in terms of treatment duration as follows: the patients who were under HCQ treatment for $\leq 5$ years or $>5$ years.

The main outcome measure of the study was OCTA parameters of the patients who were under HCQ treatment.

\section{Statistical methods}

Sample size calculations were done using the G*Power 3 software (Kiel, Germany). Sample size power was calculated for each of the evaluated OCTA parameters, and a minimum total sample of 16 patients was required to detect the differences between the control and treatment groups of the study with $80 \%$ power and $\alpha=0.05$. Categorical variables were presented as numbers and percentages, while numerical variables were expressed as the mean and standard deviation. Kolmogorov-Smirnov test was used to analyze normality of the data, and the distribution of the data was found to be normal; therefore, independent sample $t$ test was used to compare the numerical variables between the two groups. Categorical variables were compared using Chi-square test. Pearson test was used for correlation analysis. A p value $<$ 0.05 was considered statistically significant.

\section{Results}

A total of 102 eyes of 102 patients were included. There were 70 patients in the treatment group and 32 patients in the control group. The patients in the treatment group were using HCQ for a mean of $73.2 \pm 44.5$ months (ranging between 12 and 180 months). The mean age was $49.2 \pm 10.5$ years (ranging between 24 and 77 years) in the study and $48.1 \pm 16.1$ years (ranging between 20 and 69 years) in the control groups, respectively $(\mathrm{p}=0.7)$. Fiftythree $(52 \%)$ patients had rheumatoid arthritis, 21 (20.6\%) had Sjogren's syndrome, and 28 (27.5\%) had systemic lupus erythematosus.

The mean values for the OCTA parameters are summarized in Table 1. All of the vascular density values were similar between the study and treatment groups ( $p>0.05$ for all). However, the superficial whole thickness, superficial parafoveal thickness, superficial perifoveal thickness, deep whole thickness, deep parafoveal thickness, and deep perifoveal thickness were lower in the treatment group than the control group ( $p<0.05$ for all).

Twenty-nine patients were under HCQ treatment for $\leq 5$ years, and 41 patients were under HCQ treatment for $>5$ years (Table 2). The mean duration of HCQ use was $29.7 \pm 14.3$ months in the subgroup of patients who were under HCQ treatment for $\leq 5$ year and $102.2 \pm 32.3$ months in the group of patients who were under HCQ treatment for $>5$ years. The mean daily dose of HCQ was $221 \pm 41 \mathrm{mg}$ in the patients who were under treatment for $\leq 5$ years and $228 \pm 45 \mathrm{mg}$ in the patients who were under treatment for $>5$ years $(p=0.5)$. Choriocapillaris flow area, superficial whole density, and superficial perifoveal density values were different between patients who were under HCQ treatment for $\leq 5$ years and $>$ 5 years $(p<0.05$ for all). Also, superficial whole thickness, superficial parafoveal thickness, superficial perifoveal thickness, deep whole thickness, deep parafoveal thickness, and deep perifoveal thickness were lower in patients who were under HCQ treatment for $>5$ years than those who were under HCQ treatment for $\leq 5$ years $(p<0.05$ for all $)$.

\section{Discussion}

In this study, we evaluated the patients who were using HCQ for the treatment of several autoimmune diseases via OCTA. We first divided the patients into two groups as the control and treatment groups. Then, we further divided the treatment group into two subgroups according to HCQ use duration $(\leq 5$ years vs. $>5$ years). The vascular density values were similar between the control and treatment groups. However, in line with previous OCT studies [18-21], the thickness values of the treatment group were statistically lower than the control group. We observed some interesting outcomes when we divided the patients into two subgroups depending on the HCQ treatment duration. Superficial whole density and perifoveal density were higher in the subgroup of patients who were using HCQ for $\leq 5$ years. Also, most of the thickness values showed difference between the two subgroups and were better in the patients who were using $\mathrm{HCQ}$ for $\leq 5$ years. 
Table 1 The optical coherence tomography angiography outcomes of the two study groups
$F A Z$ foveal avascular zone

$* p<0.05$. Bold values represent the variables which shows statistical significance

Table 2 The optical coherence tomography outcomes of the two subgroups of treatment group

$H C Q$ hydroxychloroquine; $F A Z$ foveal avascular zone $* p<0.05$. Bold values represent the variables which shows statistical significance

\begin{tabular}{lccc}
\hline & Control group, & Treatment group, & $p$ \\
& 32 eyes & 70 eyes & \\
\hline FAZ area $\left(\mathrm{mm}^{2}\right)$ & $0.29 \pm 0.09$ & $0.31 \pm 0.11$ & 0.5 \\
FAZ perimeter $(\mathrm{mm})$ & $2.12 \pm 0.37$ & $2.16 \pm 0.43$ & 0.6 \\
Flow area (outer retina, $\left.\mathrm{mm}^{2}\right)$ & $0.86 \pm 0.55$ & $0.73 \pm 0.46$ & 0.2 \\
Flow area (choriocapillaris, $\left.\mathrm{mm}^{2}\right)$ & $2.06 \pm 0.14$ & $2.06 \pm 0.14$ & 0.9 \\
Superficial whole density $(\%)$ & $51.1 \pm 2.2$ & $49.7 \pm 3.8$ & 0.06 \\
Superficial whole thickness $(\mu \mathrm{m})$ & $\mathbf{2 8 7} \pm \mathbf{8}$ & $\mathbf{2 7 9} \pm \mathbf{1 2}$ & $\mathbf{0 . 0 0 2}$ \\
Superficial foveal density $(\%)$ & $19.7 \pm 6.2$ & $18.1 \pm 7.6$ & 0.2 \\
Superficial foveal thickness $(\mu \mathrm{m})$ & $245 \pm 23$ & $240 \pm 19$ & 0.2 \\
Superficial parafoveal density $(\%)$ & $53.1 \pm 2.9$ & $52.1 \pm 4.5$ & 0.2 \\
Superficial parafoveal thickness $(\mu \mathrm{m})$ & $\mathbf{3 2 2} \pm \mathbf{1 1}$ & $\mathbf{3 1 5} \pm \mathbf{1 6}$ & $\mathbf{0 . 0 2} *$ \\
Superficial perifoveal density $(\%)$ & $51.8 \pm 2.5$ & $50.4 \pm 3.9$ & 0.06 \\
Superficial perifoveal thickness $(\mu \mathrm{m})$ & $\mathbf{2 8 5} \pm \mathbf{8}$ & $\mathbf{2 7 8} \pm \mathbf{1 3}$ & $\mathbf{0 . 0 0 4}$ \\
Deep whole density $(\%)$ & $49.8 \pm 5.5$ & $49.7 \pm 6.2$ & 0.8 \\
Deep whole thickness $(\mu \mathrm{m})$ & $\mathbf{2 8 7} \pm \mathbf{8}$ & $\mathbf{2 7 9} \pm \mathbf{1 2}$ & $\mathbf{0 . 0 0 2 *}$ \\
Deep foveal density $(\%)$ & $36.0 \pm 6.5$ & $34.2 \pm 8.1$ & 0.2 \\
Deep foveal thickness $(\mu \mathrm{m})$ & $245 \pm 23$ & $240 \pm 19$ & 0.2 \\
Deep parafoveal density $(\%)$ & $54.2 \pm 4.9$ & $54.0 \pm 4.5$ & 0.8 \\
Deep parafoveal thickness $(\mu \mathrm{m})$ & $\mathbf{3 2 2} \pm \mathbf{1 1}$ & $\mathbf{3 1 5} \pm \mathbf{1 6}$ & $\mathbf{0 . 0 3 *}$ \\
Deep perifoveal density $(\%)$ & $51.3 \pm 5.7$ & $50.8 \pm 6.7$ & 0.7 \\
Deep perifoveal thickness $(\mu \mathrm{m})$ & $\mathbf{2 8 5} \pm \mathbf{8}$ & $\mathbf{2 7 8} \pm \mathbf{1 3}$ & $\mathbf{0 . 0 0 4 *}$ \\
\hline & & &
\end{tabular}

\begin{tabular}{|c|c|c|c|}
\hline HCQ treatment duration & $\begin{array}{l}\leq 5 \text { years } \\
29 \text { eyes }\end{array}$ & $\begin{array}{l}>5 \text { years, } \\
41 \text { eyes }\end{array}$ & $p$ \\
\hline FAZ area $\left(\mathrm{mm}^{2}\right)$ & $0.33 \pm 0.14$ & $0.30 \pm 0.09$ & 0.2 \\
\hline FAZ perimeter $(\mathrm{mm})$ & $2.19 \pm 0.54$ & $2.15 \pm 0.34$ & 0.6 \\
\hline Flow area (outer retina, $\mathrm{mm}^{2}$ ) & $0.76 \pm 0.47$ & $0.71 \pm 0.46$ & 0.6 \\
\hline Flow area (choriocapillaris, $\mathrm{mm}^{2}$ ) & $2.01 \pm 0.15$ & $2.09 \pm 0.12$ & $0.01 *$ \\
\hline Superficial whole density (\%) & $51.3 \pm 3.6$ & $48.7 \pm 3.6$ & $0.005 *$ \\
\hline Superficial whole thickness $(\mu \mathrm{m})$ & $290 \pm 7$ & $272 \pm 8$ & $>0.0001 *$ \\
\hline Superficial foveal density (\%) & $19.1 \pm 9.9$ & $17.4 \pm 5.6$ & 0.3 \\
\hline Superficial foveal thickness $(\mu \mathrm{m})$ & $245 \pm 24$ & $237 \pm 14$ & 0.1 \\
\hline Superficial parafoveal density (\%) & $52.9 \pm 4.2$ & $51.6 \pm 4.7$ & 0.2 \\
\hline Superficial parafoveal thickness $(\mu \mathrm{m})$ & $327 \pm 12$ & $308 \pm 13$ & $<0.0001 *$ \\
\hline Superficial perifoveal density (\%) & $52.0 \pm 3.8$ & $49.3 \pm 3.7$ & $0.004 *$ \\
\hline Superficial perifoveal thickness $(\mu \mathrm{m})$ & $290 \pm 8$ & $270 \pm 9$ & $<0.0001 *$ \\
\hline Deep whole density $(\%)$ & $50.6 \pm 6.5$ & $49.0 \pm 6.0$ & 0.3 \\
\hline Deep whole thickness $(\mu \mathrm{m})$ & $290 \pm 7$ & $272 \pm 8$ & $<0.0001 *$ \\
\hline Deep foveal density (\%) & $35.3 \pm 10.5$ & $33.4 \pm 6.0$ & 0.3 \\
\hline Deep foveal thickness $(\mu \mathrm{m})$ & $244 \pm 24$ & $237 \pm 14$ & 0.1 \\
\hline Deep parafoveal density $(\%)$ & $54.2 \pm 5.3$ & $53.9 \pm 4.0$ & 0.8 \\
\hline Deep parafoveal thickness $(\mu \mathrm{m})$ & $327 \pm 12$ & $308 \pm 13$ & $<0.0001 *$ \\
\hline Deep perifoveal density (\%) & $51.8 \pm 6.8$ & $50.1 \pm 6.6$ & 0.3 \\
\hline Deep perifoveal thickness $(\mu \mathrm{m})$ & $290 \pm 8$ & $270 \pm 9$ & $<0.0001 *$ \\
\hline
\end{tabular}


The exact mechanism of HCQ retinal toxicity is still debatable. A possible mechanism is the inhibition of uptake of all-trans-retinol leading to a negative effect on the visual cycle [22]. Chloroquine was detected in pigmented ocular diseases in rhesus monkeys after a long-term use [23]. The accumulation in RPE cells seems to be important as the retinal toxicity might continue even after the cessation of the drug $[3,24]$. The parafoveal and perifoveal regions are the two most common sites vulnerable to the HCQ toxicity [25]. Fovea is generally affected later. There is an ongoing debate regarding the primary site of the toxicity. Some studies suggest RPEs might be the primary affected tissue; some other suggest the outer retina/photoreceptor complex as the primary affected tissue $[3,25]$. Screening guidelines are very important in the follow-up examinations of the patients who are under HCQ treatment. The latest guidelines suggest the physicians to screen the patients via routine ophthalmologic examination, central visual field testing, OCT, and sometimes fundus autofluorescence imaging [10]. Optical coherence tomography is a very useful tool in the screening examinations of the patients using HCQ [3]. Structural OCT was widely studied to reveal the early findings of HCQ toxicity, and now OCTA, another noninvasive tool that has been used to show the retinal circulation, is being studied to detect early HCQ toxicity. In two previous studies, it was revealed that the inner and outer retinal layer was significantly thinner in the group of patients who were under HCQ [18, 26]. In concordance with these studies, we found that both superficial thickness and deep retinal thickness were decreased in the group of patients who were under HCQ treatment. In detail, the perifoveal and parafoveal regions were especially affected from the treatment and showed significant thinning. OCTA studies regarding the changes in vascular density in patients who are under HCQ treatment showed variable outcomes [13-16]. The retinal thickness decrease is a common finding in all of the OCT-based studies; however, there is not a consensus in the differences in vascular density detected via OCTA. Goker et al. showed that the vascular density in fovea in superficial and deep capillary plexus was significantly lower in patients who were under HCQ treatment than the patients who were not [14]. As a well-known fact, the first changes in HCQ toxicity begin from the perifoveal and parafoveal regions; therefore, if some change is expected in vascular density, it should be in this area. In our study, the vessel density parameters did not show statistically significant difference between the patients who were under HCQ treatment or not. But our subgroup analysis showed that the perifoveal density and parafoveal density in both superficial and deep capillary plexuses were decreased in the group of patients who were under HCQ treatment for more than 5 years. Also, Goker et al. reported increase in FAZ perimeter and FAZ area in superficial capillary plexus and full retinal vasculature which was postulated to be associated with the accumulation of the drug in the RPE cells. Forte et al. compared the OCTA findings between the patients who were under HCQ treatment for $>5$ years (20 eyes of 10 patients) and a control group (36 eyes of 18 healthy volunteer) [15]. They reported that the vascular density in fovea in deep capillary plexus and choriocapillaris was decreased. Again, the main affected zone was reported to be central fovea in this study. Bulut et al. conducted a study to evaluate the additional benefit of OCTA in the screening of HCQ-induced retinal alterations [13]. They divided the patients into two groups as low risk (receiving HCQ for $<5$ years) and high risk (receiving HCQ for $>5$ years). This study also showed us some controversial outcomes. Surprisingly, the thickness results did not show statistical difference between the low-risk and high-risk groups. Foveal, parafoveal, and perifoveal retina thickness values were similar, and only choroidal thickness was found to be decreased in the group of high-risk patients. In contrast to thickness outcomes, all of the vascular density parameters (whole, superficial, deep and foveal, parafoveal, perifoveal) were found to be decreased in the high-risk group of patients. Ozek et al. evaluated the OCTA findings in rheumatoid arthritis patients receiving HCQ treatment [16]. The authors divided the study population into three groups: patients who received HCQ for $>5$ years (24 patients), those who received $\mathrm{HCQ}$ for $<5$ years (16 patients), and healthy volunteers ( 20 patients). This study had also controversial outcomes. Nearly none of the retinal thickness outcomes differed between the three study groups except for inferior superficial plexus and hemi-inferior deep capillary plexus thickness values which were lower in the group of patients who were receiving HCQ treatment for $>5$ years. The vessel density analysis showed some positive outcomes, and deep temporal and hemi-inferior vascular density was found 
to be decreased in patients who were receiving HCQ treatment for $>5$ years. In summary, only some OCTA studies were able to detect retinal alterations in patients receiving HCQ treatment [13-16]. It seems that retinal thickness decreases both topographically and tomographically after HCQ treatment. Retinal circulation might also be affected by some means. In our study, we found that, after HCQ use, the retinal thickness was decreased. Additionally, superficial parafoveal density and perifoveal vascular density were decreased in patients who were under HCQ treatment for $>5$ years as compared to those who were under HCQ treatment for $\leq 5$ years. The blood supply of inner retinal layers comes from superficial retinal circulation [27]. The OCTA outcomes of our study might point to a possible pathologic process underlying early HCQ toxicity in the inner retinal circulation. In line with our results showing superficial retinal circulation impairment in the patients who were under HCQ treatment for $>5$ years, some data suggest that the earliest changes following HCQ exposure occur in the cytoplasm of ganglion cells and photoreceptors. The RPE seems to be affected later with the binding of the drug to melanin. Hydroxychloroquine might also influence the retinal cell's metabolism and can cause slowly the chronic toxic effects $[19,23]$. Rosenthal et al. have performed an animal study on Rhesus monkeys [23]. They have evaluated the chloroquine toxicity administered 5 days a week for as long as 4.5 years. They have found that chloroquine caused an initial toxic effect on ganglion cells and photoreceptors which was followed by alterations in choroid and RPE. In line with this animal study data, Kan et al. demonstrated a significant thinning in the macular ganglion cells and inner plexiform layer complex via OCT in patients who received HCQ treatment for at least 5 years and who had a normal VF testing [19].

The main limitation of our study was its crosssectional design. A well-conducted longitudinal study will be essential to clarify the usefulness of OCTA in HCQ retinal toxicity. The most important strength of our study was the good number of patients. We divided our HCQ using patients into two subgroups and revealed some positive outcomes regarding superficial vascular plexus density values.

In conclusion, our results indicated a decrease in the superficial capillary plexus at the parafoveal and perifoveal regions in patients were receiving HCQ treatment for $>5$ years. The OCTA imaging may thus be beneficial indirectly in the early detection of HCQ toxicity which is mainly located in the inner retinal layers such as ganglion cells. Our findings need to be confirmed in longitudinal studies.

Acknowledgement This cross-sectional study was not supported by any company. None of the authors has financial or proprietary interests in any material or method mentioned. This data has not been previously published.

Author contribution HNT, AO and UY were involved in design and conduct of the study and preparation and review of the study; HNT and UY contributed to data collection; and AO was involved in statistical analysis.

\section{Compliance with ethical standards}

Conflict of interest None of the authors has any conflicts of interest or competing interests regarding the materials used in the research.

Ethical approval All procedures performed in studies involving human participants were in accordance with the ethical standards of the institutional and/or national research committee and with the 1964 Helsinki declaration and its later amendments or comparable ethical standards.

Informed consent Informed consent was obtained from all individual participants included in the study.

\section{References}

1. Monzavi SM, Alirezaei A, Shariati-Sarabi Z, Afshari JT et al (2018) Efficacy analysis of hydroxychloroquine therapy in systemic lupus erythematosus: a study on disease activity and immunological biomarkers. Inflammopharmacol 26:1175-1182

2. Hanaoka H, Lida H, Kiyokawa T, Takakuwa Y, Kawahata K (2019) Glucocorticoid, immunosuppressant, hydroxychloroquine monotherapy, or no therapy for maintenance treatment in systemic lupus erythematosus without major organ manifestations. Clin Rheumatol 38:2785-2791

3. Yusuf IH, Sharma S, Luqmani R, Downes SM (2017) Hydroxychloroquine retinopathy. Eye (Lond) 31:828-845

4. Gao J, Hu S (2020) Update on use of chloroquine/hydroxychloroquine to treat coronavirus disease 2019 (COVID19). Biosci Trends. https://doi.org/10.5582/bst.2020.03072 [Epub ahead of print]

5. Bernstein HN (1991) Ocular safety of hydroxychloroquine. Ann Ophthalmol 23:292-296

6. Melles RB, Marmor MF (2014) The risk of toxic retinopathy in patients on long-term hydroxychloroquine therapy. JAMA Ophthalmol 132:1453-1460

7. Wetterholm DH, Winter FC (1964) Histopathology of chloroquine retinal toxicity. Arch Ophthalmol 71:82-87 
8. Korthagen NM, Bastiaans J, van Meurs JC, van Bilsen K, van Hagen PM, Dik WA (2015) Chloroquine and hydroxychloroquine increase retinal pigment epithelial layer permeability. J Biochem Mol Toxicol 29:299-304

9. Marmor MF, Kellner U, Lai TY, Lyons JS, Mieler WF (2011) Revised recommendations on screening for chloroquine and hydroxychloroquine retinopathy. Ophthalmology 118:415-422

10. Marmor MF, Kellner U, Lai TY, Melles RB, Mieler WF (2016) Recommendations on screening for chloroquine and hydroxychloroquine retinopathy (2016 revision). Ophthalmology 123:1386-1394

11. Kellner U, Renner AB, Tillack H (2006) Fundus autofluorescence and mfERG for early detection of retinal alterations in patients using chloroquine/hydroxychloroquine. Invest Ophthalmol Vis Sci 47:3531-3538

12. Tsang AC, Ahmadi Pirshahid S, Virgili G, Gottlieb CC, Hamilton J, Coupland SG (2015) Hydroxychloroquine and chloroquine retinopathy: a systematic review evaluating the multifocal electroretinogram as a screening test. Ophthalmology 122:1239-1251

13. Bulut M, Akıdan M, Gözkaya O, Erol MK, Cengiz A, Çay HF (2018) Optical coherence tomography angiography for screening of hydroxychloroquine-induced retinal alterations. Graefes Arch Clin Exp Ophthalmol 256:2075-2081

14. Goker YS, Ucgul Atılgan C, Tekin K, Kizıltoprak H et al (2019) The validity of optical coherence tomography angiography as a screening test for the early detection of retinal changes in patients with hydroxychloroquine therapy. Curr Eye Res 44:311-315

15. Forte R, Haulani H, Dyrda A, Jürgens I (2019) Swept source optical coherence tomography angiography in patients treated with hydroxychloroquine: correlation with morphological and functional tests. Br J Ophthalmol. https:// doi.org/10.1136/bjophthalmol-2018-313679 [Epub ahead of print]

16. Ozek D, Onen M, Karaca EE, Omma A, Kemer OE, Coskun C (2019) The optical coherence tomography angiography findings of rheumatoid arthritis patients taking hydroxychloroquine. Eur J Ophthalmol 29(5):532-537. https://doi. org/10.1177/1120672118801125 Epub 2018 Sep 19

17. Sarwar S, Hassan M, Soliman MK, Halim MS et al (2018) Diurnal variation of choriocapillaris vessel flow density in normal subjects measured using optical coherence tomography angiography. Int J Retina Vitreous 4:37
18. Ulviye Y, Betul T, Nur TH, Selda C (2013) Spectral domain optical coherence tomography for early detection of retinal alterations in patients using hydroxychloroquine. Indian $\mathrm{J}$ Ophthalmol 61:168-171

19. Kan E, Yakar K, Demirag MD, Gok M (2018) Macular ganglion cell-inner plexiform layer thickness for detection of early retinal toxicity of hydroxychloroquine. Int Ophthalmol 38:1635-1640

20. Cakir A, Ozturan ŞG, Yildiz D, Erden B et al (2019) Evaluation of photoreceptor outer segment length in hydroxychloroquine users. Eye (Lond) 33:1321-1326

21. Allahdina AM, Chen KG, Alvarez JA, Wong WT, Chew EY, Cukras CA (2019) Longitudinal changes in eyes with hydroxychloroquine retinal toxicity. Retina 39:473-484

22. Grassmann F, Bergholz R, Mandl J, Jagle H, Ruether K, Weber BH (2015) Common synonymous variants in $\mathrm{ABCA} 4$ are protective for chloroquine induced maculopathy (toxic maculopathy). BMC Ophthalmol 25:18

23. Rosenthal AR, Kolb H, Bergsma D, Huxsoll D, Hopkins JL (1978) Chloroquine retinopathy in the rhesus monkey. Invest Ophthalmol Vis Sci 17:1158-1175

24. Turgut B, Turkcuoglu P, Serdar Koca S, Aydemir O (2009) Detection of the regression on hydroxychloroquine retinopathy in optical coherence tomography. Clin Rheumatol 28:607-609

25. de Sisternes L, Hu J, Rubin DL, Marmor MF (2015) Localization of damage in progressive hydroxychloroquine retinopathy on and off the drug: inner versus outer retina, parafovea versus peripheral fovea. Invest Ophthalmol Vis Sci 56:3415-3426

26. Eo DR, Lee MG, Ham DI, Kang SW et al (2017) Frequency and clinical characteristics of hydroxychloroquine retinopathy in Korean patients with rheumatologic diseases. J Korean Med Sci 32:522-527

27. Hagag AM, Gao SS, Jia Y, Huang D (2017) Optical coherence tomography angiography: technical principles and clinical applications in ophthalmology. Taiwan J Ophthalmol 7:115-129

Publisher's Note Springer Nature remains neutral with regard to jurisdictional claims in published maps and institutional affiliations. 